\title{
Far-Field Species Distribution Measurements on the BHT-600 Hall Thruster Cluster
}

\author{
Allen L. Victor ${ }^{1}$ and Alec D. Gallimore ${ }^{2}$ \\ University of Michigan, Ann Arbor, Michigan, 48109, USA \\ Thomas H. Zurbuchen ${ }^{3}$ \\ University of Michigan, Ann Arbor, Michigan, 48109, USA
}

Measurements via the Top Hat Electric Propulsion Plume Analyzer (TOPAZ) on the Busek BHT-600 Hall thruster cluster are presented. TOPAZ incorporates a 'top hat' design with an analyzer constant of 100 resulting in a wide energy-range capability and high angular and energy resolutions. A time-of-flight analyzer is also incorporated for mass-tocharge (and hence charge-state) discrimination from within the plume. Energy profiles measured from different regions of the cluster revealed lower-energy (i.e., energies significantly below the discharge voltage) ions emanating from positions closer to the cathode, while higher-energy (i.e., energies near or higher than the discharge voltage) ions were measured along the discharge channel centerlines. Low-energy ions were also measured from behind the cathodes only during cluster operation indicating a possible cross-pollination of the cathode plume with the opposite thruster. Multiply-charged xenon ions (i.e., $\mathrm{Xe}^{3+}$ and $\mathrm{Xe}^{4+}$ ) were measured for $0^{\circ}$ and $10^{\circ}$ plume angles while the $20^{\circ}$ plume angle revealed mostly singly- and doubly-charged xenon. Calculations of the axial and radial velocity distributions for the first three charge-states downstream of the cluster centerline revealed a symmetric triple-peak structure in the radial velocity distributions and a doublepeak profile in the axial velocity distribution of the first charge-state of xenon.

\section{Nomenclature}

\begin{tabular}{|c|c|c|}
\hline$E$ & $=$ particle energy & $\theta \quad=$ aperture angle \\
\hline$K$ & $=$ analyzer constant & $=$ electric potential \\
\hline$R_{1}$ & $=$ deflection plate gap radius & \\
\hline$R_{2}$ & $=$ grounded plate gap radius & \\
\hline$R_{3}$ & $=$ top hat radius & \\
\hline$R_{C}$ & $=$ gap centerline radius & \\
\hline$R_{G}$ & $=$ guiding plate radius & \\
\hline$R_{P}$ & $=$ particle radius of motion & \\
\hline$S$ & $=$ aperture radius & \\
\hline$V_{D}$ & $=$ deflection plate voltage & \\
\hline$d_{\text {gate }}$ & $=$ gate distance & \\
\hline$d_{\text {TOF }}$ & $=$ time-of-flight distance & \\
\hline$m$ & $=$ particle mass & \\
\hline$q$ & $=$ charge of particle & \\
\hline$t_{T O F}$ & $=$ particle time-of-flight & \\
\hline$v$ & $=$ velocity & \\
\hline$\Delta R$ & $=$ gap distance & \\
\hline$\Delta x$ & $=$ distance between exit of gap and particle detector & \\
\hline$\alpha$ & $=$ elevation angle & \\
\hline$\beta$ & $=$ azimuthal angle of incoming particles & \\
\hline
\end{tabular}

\footnotetext{
${ }^{1}$ Former Graduate Student, Department of Aerospace Engineering, allen.victor@gmail.com.

${ }^{2}$ Arthur F. Thurnau Professor, Department of Aerospace Engineering, alec.gallimore@umich.edu.

${ }^{3}$ Professor, Department of Aerospace Engineering, thomasz@umich.edu.
} 


\section{Introduction}

$\mathrm{E}^{\mathrm{s}}$ LECTRIC propulsion (EP) offers power-efficient, high specific impulse $\left(I_{s p}\right)$ options for deep-space missions as well as station keeping, orbital transfer, and attitude control requirements for near-Earth spacecraft. Hall thrusters are a type of EP system that utilizes electric and magnetic fields to produce thrust. Electrons emitted by a cathode travel upstream towards a positively-charged anode. A magnetic field that is applied in the perpendicular direction of the electric field hinders electron motion and creates a closed electron drift region. Propellant (e.g., xenon or krypton) is injected at the anode of an annular discharge channel, and ionized through collisions with the electrons caught in the closed electron drift region. The magnetic field has very little effect on the relatively massive ions. The electric field, however, accelerates the ions downstream away from the anode producing thrust. Currently, mid-power Hall thrusters achieve specific impulses between 1500-2500 seconds and electrical power efficiencies between $50-60 \%{ }^{1}$

Recent trends in Hall thruster research by the USAF and US industry have included the high-power $(>20 \mathrm{~kW})$ regime. ${ }^{2}$ NASA is sponsoring high-power / high- $\mathrm{I}_{\mathrm{sp}}(10 \mathrm{~kW} />=2000 \mathrm{~s})$ Hall thruster technology through the NASA Glenn Research Center (GRC). ${ }^{3}$ The NASA-457 Hall Thruster developed at GRC produced the highest power level and thrust $(75 \mathrm{~kW}$ and $2.9 \mathrm{~N})$ achieved by any xenon-propellant Hall Thruster. ${ }^{4}$ High I $_{\mathrm{sp}}$ anode layer type (TAL) Hall thrusters have achieved specific impulses above $4100 \mathrm{~s}$ at this center as well. ${ }^{5}$

For these high-powered engines and future even more powerful engines being developed, plume characterization is imperative for determining their effect on spacecraft systems. Plasma transport properties, ionic charge state, and ion energy distributions are also important for understanding how Hall thrusters work and for improving their performance. $^{6}$

One technique for determining the energy-to-charge distribution of plasma is to use an electrostatic analyzer. A specific geometry for the electrostatic analyzer, which allows for a wide field-of-view, is the top hat analyzer. This electrostatic analyzer consists of a sphere and a concentric shell with an aperture at the apex of the outer shell. The inner sphere is set to a specific voltage to allow for a narrow energy band of particles to pass through the aperture. By virtue of its geometry, the top hat analyzer is capable of having a 360-degree azimuthal field-of-view. Steering electric fields above the aperture allow for a field-of-view in the vertical direction as well. Structural constraints, however, diminish the total field-of-view in both directions.

The mass-per-charge of ions can also be determined through several methods. Magnetic sectors, quadrupole mass filters, and time-of-flight mass spectrometers are common techniques employed to determine the mass distribution of charged particles. The Top Hat Electric Propulsion Plume Analyzer (TOPAZ) incorporates a time-offlight design to reveal the mass-per-charge spectrum of the plasma of interest. The velocity distribution function (VDF) within the field-of-view can be extracted from mass-per-charge measurements in conjunction with energyangular measurements from TOPAZ

Previous measurements on the BHT-600 cluster via TOPAZ have yielded interesting energy-angle correlations in the far-field plume. Ions emanating from near the cathode tend to have slightly lower energies than those from other parts of the cluster. ${ }^{7}$ The aim of this study is to ascertain the individual relationships of the multiple charge states of the propellant with the energy-angle correlation.

The motivation for the design of the TOPAZ is first discussed. The theory of operation for the energy analyzer and time-of-flight mass spectrometer is then presented, and the relevant design parameters are derived and related to the performance of the instrument.

\section{Design Motivation}

Electrostatic analyzers have been and are currently employed on spacecraft to investigate space plasmas such as solar wind as well as the ionospheres and magnetospheres of Earth and other planets. ${ }^{8,9}$ Space plasmas offer a wide range of particle energies from less than $1 \mathrm{eV}$ to several $\mathrm{MeV}$. This has led to design of electrostatic analyzers capable of detecting particles over several orders of magnitude in energy ${ }^{8}$ however, these types of plasmas have an ion number density several orders of magnitude lower than Hall thruster and ion engine plume.

Figure 1 describes the typical energy and number density ranges of space, laboratory, and Hall thruster and ion engine plasmas. The Hall thruster plume, the plasma of interest for TOPAZ, is nestled between laboratory plasmas (glow discharges and fusion experiments) and space plasmas (solar wind and the magnetotail) on the density scale. The energy range between Hall thruster plume and magnetotail plasma are similar. The primary difference between these two plasmas is the number density for the Hall thruster plume is several orders of magnitude greater. 
There are many examples space plasma diagnostics through top hat analyzers. EP plume measurements with this type of device, however, are much rarer. The Plasma Experiment for Planetary Exploration (PEPE), flown on Deep Space 1 (DS1), included a dual top hat analyzer used to measure electrons and ions from the solar wind, spacecraft photoelectron sheath, and products of the xenon ion propulsion system. Low-energy xenon ions $(<40 \mathrm{eV})$ created from the beam ion interaction with neutral xenon particles were observed by PEPE. ${ }^{13}$

Although beam ions were not measured by PEPE due to the position and orientation of the thruster with respect to the instrument, the observation of chargeexchange ions provides evidence for the top hat analyzer as a plume diagnostics tool for measuring facility affects. Hall thrusters have been shown to

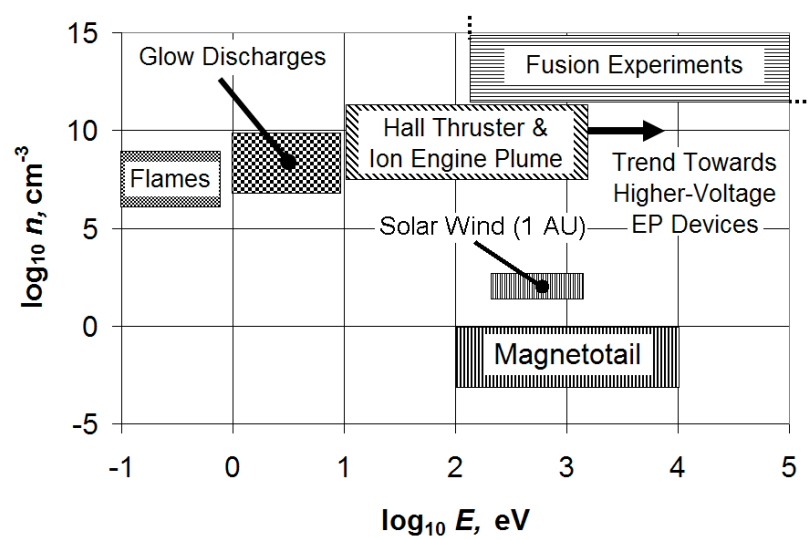

Figure 1. Number density and energy of typical space, laboratory, and electric propulsion plasmas. ${ }^{8,10-12}$ yield higher current density profiles in the far-field plume at higher background pressures. It is theorized that charge-exchange ions created from beam ions and neutral background particles are the culprit for the elevated current densities. ${ }^{14}$ Diagnostic tools capable of characterizing the low energy charge-exchange ions as well as the high energy beam ions are therefore necessary to distinguish facility effects on plume diagnostics.

Charge states of propellant ions affect the charge-utilization efficiency, and hence the overall performance of Hall thrusters. ${ }^{15}$ An accurate measurement of the presence of multiple-charge states of propellant ions through a mass analyzer will provide an in-situ estimation of the charge-utilization efficiency. The time-of-flight technique utilized by TOPAZ for mass spectroscopy is utilized, and the measurements in the BHT-600 Hall thruster cluser are presented in this paper.

\section{Analyzer Design}

The TOPAZ design theory is discussed in the following section. The design requirements for the analyzer are first described. SIMION, an ion trajectory code, was used to predict the angular and energy resolutions for TOPAZ. The final design for the analyzer was determined through an iterative process with SIMION. This is described in more detail in Ref. 16.

\section{A. Design Requirements}

An increase in power is expected for EP thruster development, and the acceleration potentials (and hence beam ion energies) are expected to increase in the foreseeable future. While Hall thruster voltages are not expected to increase above $2 \mathrm{keV}$, ion thrusters that are being considered for deep-space missions may have xenon ion beams of energy above $6 \mathrm{keV} \cdot{ }^{17} \mathrm{~A} \mathrm{Xe}^{2+}$ ion accelerates to $12-14 \mathrm{keV}$ at this potential; hence TOPAZ has been designed to have a high energy measurement capability up to $15 \mathrm{keV}$. The nature of a top hat analyzer allows for the lower bound to be close to $0 \mathrm{eV}$, since the plate potentials correspond directly with the measured energy. The lower energy bound therefore is set by the accuracy of the power supplies used.

Since TOPAZ is a far-field plume diagnostics instrument, an adequate field of view of the thruster is required to "image" the ions projected from the entire discharge channel. A $30^{\circ}$ vertical field-of-view allows for $57.7 \mathrm{~cm}$ of an object to be viewed from $1 \mathrm{~m}$ away, well within the size range of most thrusters. The azimuthal field-of-view is ideally $360^{\circ}$, but structural constraints diminish this to $112^{\circ}$. The angular resolutions of the field of view are within $2^{\circ} \times 2^{\circ}$ for the vertical and azimuthal directions, respectively. This resolution provides enough accuracy to determine a detected ion from the discharge channel, a different part of the thruster, or from the plume.

An adequate mass-per-charge resolution of TOPAZ is desired for detecting multiple-charge states (up to 4+) of propellants (e.g., Xenon and Krypton) and background ions in the chamber (e.g., $\mathrm{N}^{+}$and $\mathrm{O}^{+}$). This allows TOPAZ to directly measure the charge-utilization efficiency of the thruster, as well as characterize the ingestion of background particles by the thruster inducing facility effects.

\section{B. Theory of Operation}

Since TOPAZ incorporates both energy analyzer and mass spectrometer components, a theoretical discussion of both follows. 


\section{Electrostatic Energy Analysis}

The top hat analyzer utilizes a radial electric field to guide ions through a spherical shell-shaped channel between a grounded plate and a negatively charged deflection plate. Figure 2 displays the key dimensions and plates for a top hat analyzer. The most important criterion is the ratio of the channel radius $R_{C}$ to the gap distance $\Delta R$ $\left(\Delta R=R_{2}-R_{l}\right)$ which sets the analyzer constant (Eq. 1).

$$
K \equiv \frac{R_{C}}{\Delta R}
$$

The analyzer constant $K$ determines the energy resolution, energy-to-voltage ratio, and other properties of the analyzer. The channel radius is simply the average of the inner and outer radii for the gap. Equating the applied force required to turn a particle at the channel radius with the electric field generated in the gap, the voltage is related to the energy-to-charge ratio. For high analyzer constants, the electric field can be assumed to be linear between the deflection plate and grounded plate. Equation (2) displays the simple relationship between the deflection plate voltage $V_{D}$, the analyzer constant, and the expected energy-to-charge ratio to be measured.

$$
\frac{E}{q}=-\frac{K V_{D}}{2}
$$

The top hat radius $R_{3}$ and the aperture angle $\theta$ determine the average elevation angle and the effective aperture area for the measured ions, respectively. The maximum ratio of detectable ions to incoming ions is

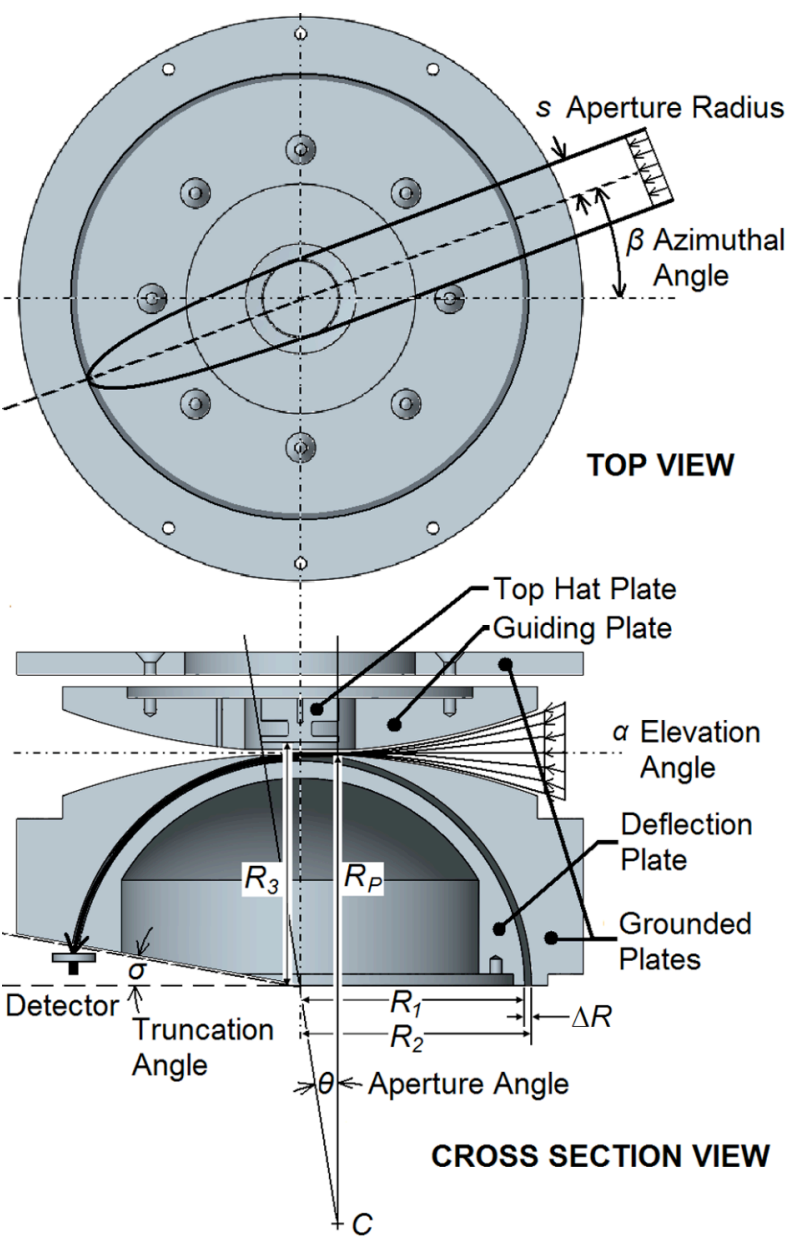

Figure 2. Principal design parameters of a top hat analyzer.

realized when the top hat radius $R_{3}$ is at least as large as the analyzer gap distance. ${ }^{18}$ This ratio yields a top hat radius of approximately $R_{3}=R_{1}+2 \Delta$.

The inner deflection plate radius $R_{I}$ is held to a negative plate potential to detect positively charged ions. The outer radius is kept at ground potential. The top hat plate is also usually held at ground, but can be biased to increase transmissivity, or used as a gating plate for time-of-flight applications.

Guiding plates, which can vary either positively or negatively in plate potential, allow for variance in the vertical angular direction (elevation angle) for the measured ions. Ions coming from the selected elevation angle are guided into the top hat region such that their entrance angle is approximately horizontal above the deflection plate.

Ideally, the aperture angle $\theta$ (in radians) is proportional to the inverse square root of the analyzer constant. ${ }^{16}$

$$
\theta \approx \sqrt{\frac{1}{K}}
$$

The top hat plate should have the same radius as the aperture, such that the field generated between the top hat and the deflection plate is half that of the field between the deflection and grounded plates. This scenario allows for an ion to follow a "grazing" trajectory with the grounded plate and arrive at the detector.

Time-of-Flight Mass Spectroscopy

Time-of-flight mass spectrometers utilize the principle that particles of different masses with the same energy $E$ travel with different velocities inversely proportional to the square root of the mass (Eq. 4). 


$$
v=\sqrt{\frac{2 E}{m}}
$$

The time-of-flight $t_{T O F}$ of the particle over a prescribed distance $d_{\text {TOF }}$ is therefore directly proportional to the square root of the mass (Eq. 5).

$$
t_{T O F}=d_{T O F} \sqrt{\frac{m}{2 E}}
$$

If the particle is an ion of charge $q$ which has traveled over a potential $\Delta \varphi$, Eq. (5) can be rewritten in terms of the mass-per-charge as a function of the time-of-flight (Eq. 6).

$$
\frac{m}{q}=2 \Delta \varphi\left(\frac{t_{T O F}}{d_{T O F}}\right)^{2}
$$

To determine the time-of-flight of a particle, the ions flow to the detector are turned "on" and "off" by use of the top hat plate which acts as an electrostatic gate. To "open" the gate, the top hat plate is biased with a positive voltage such that the transmission of ions through the channel to the detector is maximized. To "close", the voltage of the plate is biased to a negative voltage (or ground if possible). In this state, no ions are able to travel through the aperture and arrive at the detector.

The use of the top hat plate as a gate for the ion flux to the detector requires voltage pulses, such that the pulse width is long enough for the slowest ion of interest to travel across the gate. However, if the gate is biased "on" for too long, a wider range of ions traveling at different velocities (i.e., different masses of the same energy) arrive at the detector at the same time. Therefore the smallest pulse width possible is desired to maintain the best mass resolution while not sacrificing a loss in signal from the slowest (and largest mass-per-charge) ion.

Equation (7) depicts the required pulse width $t_{\text {gate }}$ required to allow the slowest ion to traverse across the gate distance $d_{\text {gate }}$.

$$
t_{\text {gate }}>d_{\text {gate }} \sqrt{\frac{m_{\max }}{2 q \varphi}}
$$

The total distance the ion travels from the entrance of the gate (the leading edge of the aperture) to the detector is expressed in Eq. (8).

$$
d_{\text {TOF }}=\frac{R_{P} \theta}{2}+\frac{\pi R_{C}}{2}+\Delta x
$$

The first term represents the flight of the ion over radius $R_{p}$ from the leading edge of the aperture entrance (see Fig. 2) to the centerline of TOPAZ over the middle of the aperture radius. The ion then travels through the channel at a radius of $R_{C}$ making a $90^{\circ}$ turn the entrance to the exit of the gap. Finally the distance between the exit of the gap and the entrance into the detector is represented as $\Delta x$.

If the particle trajectory over the aperture is assumed to be approximately twice the channel radius $R_{C}$, Eq. (8) can be rewritten in terms of the analyzer constant $K$ with help from Eq. (3).

$$
d_{T O F} \approx \frac{R_{C}}{\sqrt{K}}+\frac{\pi R_{C}}{2}+\Delta x
$$


By relating Eq. (7) and Eq. (10), the mass resolution is shown to be inversely proportional to the analyzer constant to a first order approximation.

$$
\frac{\Delta m}{m} \approx \frac{4}{\pi \sqrt{K}}
$$

The above formulation indicates that top hat analyzers with high analyzer constants $(K>50)$ are particularly well-suited for time-of-flight techniques, since the ratio of the top hat plate (gate distance) to the channel distance (time-of-flight distance) is small.

Time-of-flight measurements on an ion beam utilizing krypton and xenon propellants through TOPAZ have yielded the first four charge states for energies 1-5 keV. ${ }^{20}$ Since the BHT-600 cluster operates at a much lower voltage $(300 \mathrm{~V})$, the instruments viability for detecting these charge states in the plume has been demonstrated.

\section{Analyzer Design Specifications}

Table 1 describes the design specifications and performance parameters for TOPAZ. A high analyzer constant of 100 was chosen to meet the energy resolution requirements. The high analyzer constant also aids in providing an adequate time-offlight mass resolution. The angular resolutions and field-ofview were determined through SIMION and verified through experimental calibration. The method of the design and characterization of the electrostatics and time-of-flight of TOPAZ is discussed in further detail in previous articles. ${ }^{16,19}$

The biased and grounded plates in TOPAZ are made of

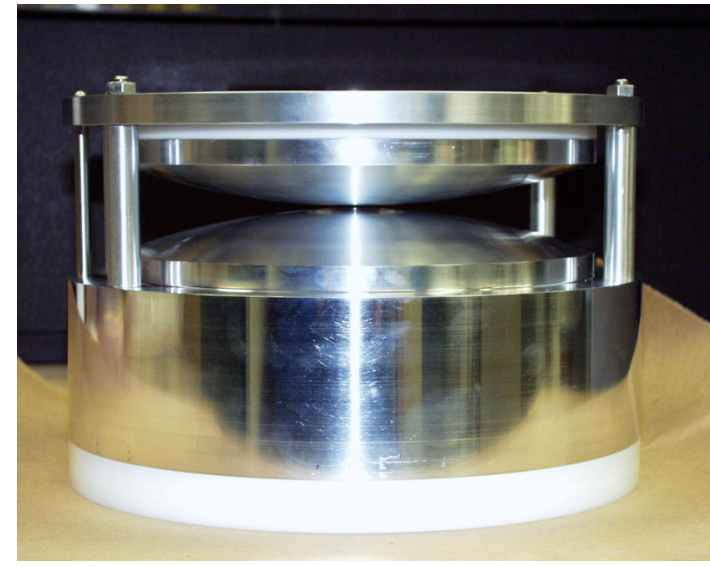

Figure 3. Final construction of TOPAZ. Aluminum 6061-T6. High-temperature Machinable Glass Mica insulators are used to position the aluminum plates of TOPAZ in the correct position. Delrin, a non-conductive polymer (polyoxymethylene), provides the required separation for the top hat plate above the aperture.

\section{Measurements on the BHT-600 Cluster}

To characterize the plume of a cluster of Hall thrusters, TOPAZ was placed one meter downstream of a cluster of four $600 \mathrm{~W}$ BHT-600 Hall thrusters (Fig. 4). These thrusters operate on xenon propellant. Measurements were conducted at thruster plume angles of $0^{\circ}$ and $60^{\circ}$ (to the left of the cluster). Each Hall thruster was operated at 300 $\mathrm{V}$ and 2 A. A similar setup utilized for the time-of-flight characterization of TOPAZ is incorporated for mass spectrometry measurements in the Large Vacuum Test Facility (LVTF) at the Plasmadynamics and Electric Propulsion Laboratory (PEPL).

\section{A. Experimental Setup}

All measurements were conducted in the University of Michigan's $6 \mathrm{~m}$ diameter by $9 \mathrm{~m}$ long LVTF at PEPL. Four model TM-1200 Re-Entrant Cryopumps are used to create an ultimate base pressure of $3 \times 10^{-7}$ Torr. The pressure for these measurements during cluster and single thruster operation were $5.0 \times 10^{-6}$ Torr and $1.96 \times 10^{-6}$ Torr, respectively. These values are corrected for xenon through equation $11 .^{20}$

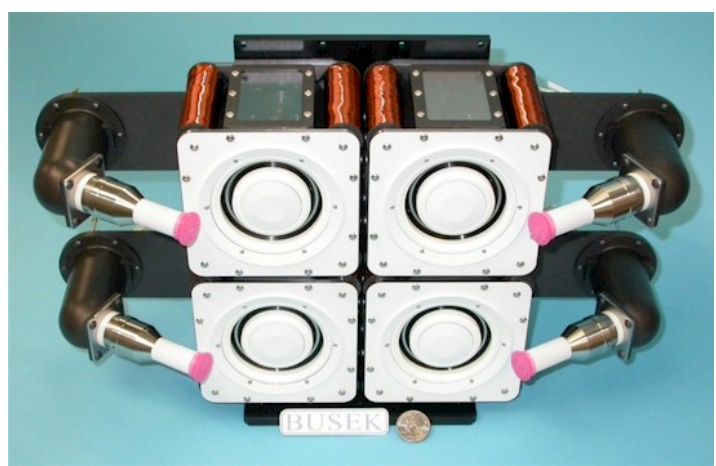

Figure 4. Cluster of four $600 \mathrm{~W}$ BHT-600 Hall thrusters. 

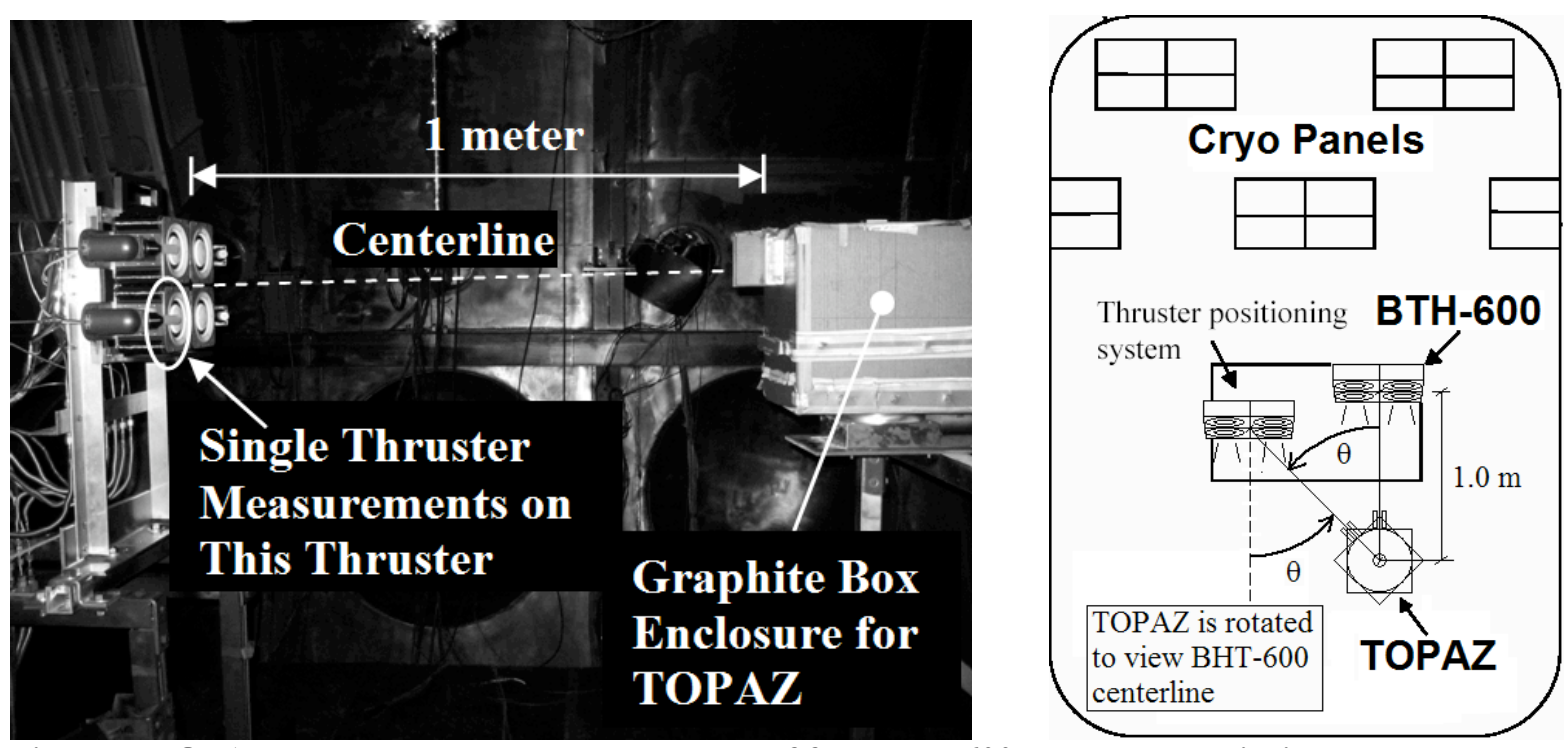

Figure 5. TOPAZ mounted one meter downstream of four BHT-600 Hall thrusters inside the LVTF.

$$
p_{c}=\frac{p_{i}-p_{b}}{2.87}+p_{b}
$$

TOPAZ was placed exactly one meter downstream of the centerline of the four BHT-600 Hall thrusters. A graphite box with a slit entrance is used to protect TOPAZ from the high energy beam flux. Figure 5 displays the BHT-600 thrusters and TOPAZ inside the LVTF. The BHT-600 cluster was acquired by PEPL for basic research on Hall thruster cluster characterization, their facility effects, and plume diagnostics. Each thruster has a $6 \mathrm{~cm}$ outside channel diameter and a centerline-to-centerline separation of $11 \mathrm{~cm}$ in the square-cluster configuration. Each thruster is operated at $300 \mathrm{~V}$ and $2 \mathrm{~A}$ with anode and cathode flow rates of $2.5 \mathrm{mg} / \mathrm{s}$ and $0.5 \mathrm{mg} / \mathrm{s}$, respectively. TOPAZ is mounted on two linear tables, which are positioned perpendicular to each other, allowing for two-dimensional movement. These tables allow for measurements by TOPAZ to be taken from different thruster angles. To measure different azimuthal angles, TOPAZ and the CEM are rotated about the centerline of the analyzer by using a Daedal 20600RT rotary table. The rotational stage has a resolution of $0.001^{\circ}$ and is operated through LabVIEW 7.1 software. A thermal couple was placed on TOPAZ to monitor the temperature of the instrument. During all tests the temperature remained between $20-55^{\circ} \mathrm{C}$.

Figure 6 describes the electrical schematic and controlling instrumentation for time-of-flight measurements within the LVTF. 


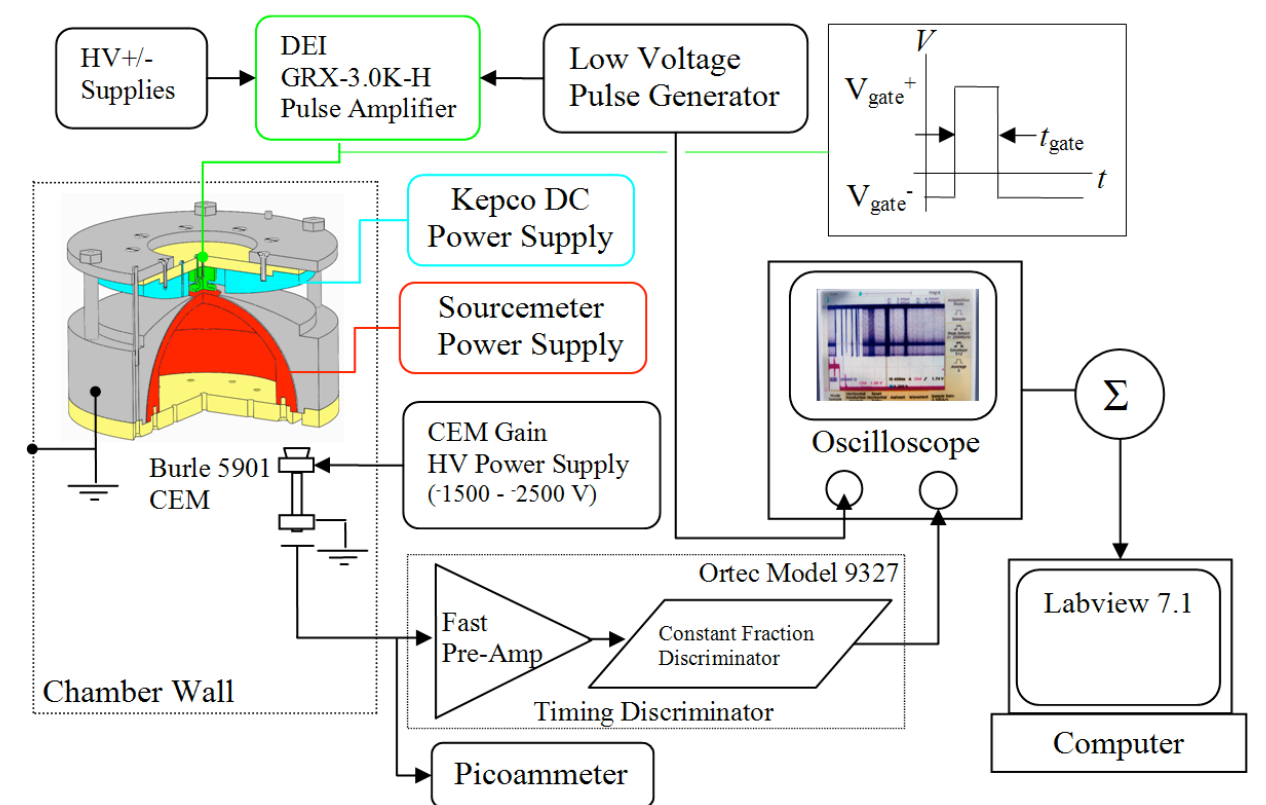

Figure 6. Schematic of electrical components utilized for time-of-flight measurements.

The energy-per-charge is determined by the voltage of the deflection plate (in red) set by the Sourcemeter power supply. The guiding plate potential (in turquoise) is controlled by a Kepco DC power supply. The top hate plate potential (in green) is set by the Directed Energy, Inc. GRX-3.0K-H the high voltage pulse amplifier. A low voltage pulse generator sets drives the pulse width and frequency, while external high-voltage power supplies set the upper and lower peak voltages $\left(V_{\text {gate }}{ }^{+}\right.$and $\left.V_{\text {gate }}{ }^{-}\right)$of the pulse. The positive voltage allows ions to flow through the aperture, while a negative voltage prevents any ions from being detected. The pulse generator sends a signal to the oscilloscope for monitoring the start time of the pulse.

A Burle 5901 channel electron multiplier (CEM) is employed to detect ions after they have passed through TOPAZ. The CEM operates by allowing ions to strike a ceramic surface with a high secondary electron emission rate. A voltage of -1500 to $-2500 \mathrm{~V}$ is placed over a distance of $3.8 \mathrm{~cm}$ which induces secondary electron multiplication within the CEM, and the signal is amplified by approximately $5 \times 10^{7}$. After an ion flies into the CEM, a pulse is created due to the avalanche of secondary electrons emitted. The gain for this response is controlled by a high-voltage power supply.

The pulse exits the chamber, and the current is converted into a voltage pulse via the fast pre-amplifier. A constant fraction discriminator shapes the pulse such that the peak voltage and width are constant. The signal is sent to the oscilloscope, and the delay of detected ions with respect to the pulse generator is measured. The voltage as a function of time is averaged over 320 samples on the oscilloscope and summed through LabVIEW code and saved on a personal computer. A Keithley picoammeter is alternately used to measure the current generated by the CEM outside of the LVTF for energy distribution measurements.

\section{B. Procedure}

Measurements are conducted from varying plume angles from $0^{\circ}-20^{\circ}$ in increments of $10^{\circ}$. Time-of-flight measurements are conducted for energies between $0-650 \mathrm{eV}$, while the azimuthal and elevation angle of ions being detected is changed through detector positioning and variation of the guiding plate potential, respectively. A single thruster as well as the full cluster is operated for comparison of their plume properties. For all measurements, the thrusters were operated for 10 minutes until stable discharge voltage and current settings were reached.

Time-of-flight (TOF) measurements are converted to mass-per-charge profiles through use of the known TOF distance of $18 \mathrm{~cm}$. Guiding and deflection plate potentials are converted into an elevation angle and energy-percharge through voltage relationships previously characterized, ${ }^{7}$ while the azimuthal angle is directly measured. A conversion from spherical to Cartesian coordinates of energy-per-charge, mass-per-charge, elevation angle, and azimuthal angle reveals the velocity distribution profile within the field of view for each of the charge states measured. 


\section{Results \& Discussion}

Measurements of the energy-per-charge and mass-per-charge allowed for the calculation of charge state and estimations of velocimetry to be calculated for the ion populations. The energy-percharge measurements are presented first with the mass-per-charge profiles and velocity distributions to follow.

\section{Energy-per-Charge Distributions}

By profiling energy profiles for separate azimuthal angles, and "image" of the energy-azimuthal angle relation is formed. For this measurement the guiding plate was grounded for an approximate measurement along the centerline of the thruster. Figure 7 displays the contour plot of this relationship.

As with the cluster operation, a plot of the energy-to-charge ratio as a function of azimuthal angle was done for the single-thruster operating condition as well. Figure 8 displays a plot of the energy-tocharge relationship with the azimuthal angle for the bottom-left thruster.

Since some important features of Fig. 7 occur with low currents, an exponential color coding is utilized to enhance these features. The cluster setup from the vintage point of TOPAZ is placed above the graph to azimuthally correlate measurements on the thruster with the plot. Since the density of data points is beyond the energy and azimuthal resolutions, the error "box" is assumed to have dimensions of these resolutions.

It is interesting to note that each energy profile (a vertical slice) is highly dependant on the azimuthal angle being measured. The bulk of the current was measured at energies between $240-325$ $\mathrm{eV}$ at positions directly in front the cluster. However, directly along the thruster centerlines, a wide energy-spread was measured between $200 \mathrm{eV}-420 \mathrm{eV}$.

As with the cluster measurement of energy-to-charge ratio as a function of azimuthal angle, a similar trend of ions with lower energies emanating from the near the cathode is noticed for the lower-left single thruster operation. In comparison with Fig. 7, the emanation area is much smaller, not just overall, but per thruster. The effective width of the detection of ions is over approximately $6^{\circ}$ which correlates to $11.8 \mathrm{~cm}$ from $112.3 \mathrm{~cm}$ downstream of the thruster (i.e., the effective plume interrogation point). This is approximately twice the discharge channel diameter. For the cluster operation, the area was over approximately $20^{\circ}$. A significant number of ions were detected from behind the cathodes, whereas for the single thruster operation these ions are absent.

A possible scenario for this is the ionization of propellant particles due to interaction with the opposite-cathode plume. Since the cathode plume likely extends across the entire cluster and over the oppositely-placed cathodes, electrons which "overshot" the discharge channels could provide an ionization source behind the oppositely placed cathodes. The cross-pollination of electrons would effectively increase the total number of ions produced per cathode. Since the overall ionization region is increased, a slight increase in thrust is predicted for the cluster configuration versus the sum of four single-thruster measurements of thrust. This scenario assumes the ions generated behind the cathodes were not "displaced" ions that would have been accelerated in front of the discharge channel.

Measurements and simulations by Beal on the BHT-200-X3 cluster, showed that the cluster configuration yields an ion-focusing effect on CEX ions while the beam ions are largely unaffected by weak fields generated with the concurrent operation of the thrusters. ${ }^{21}$ Walker measured thrust for single thruster and a two-thruster cluster

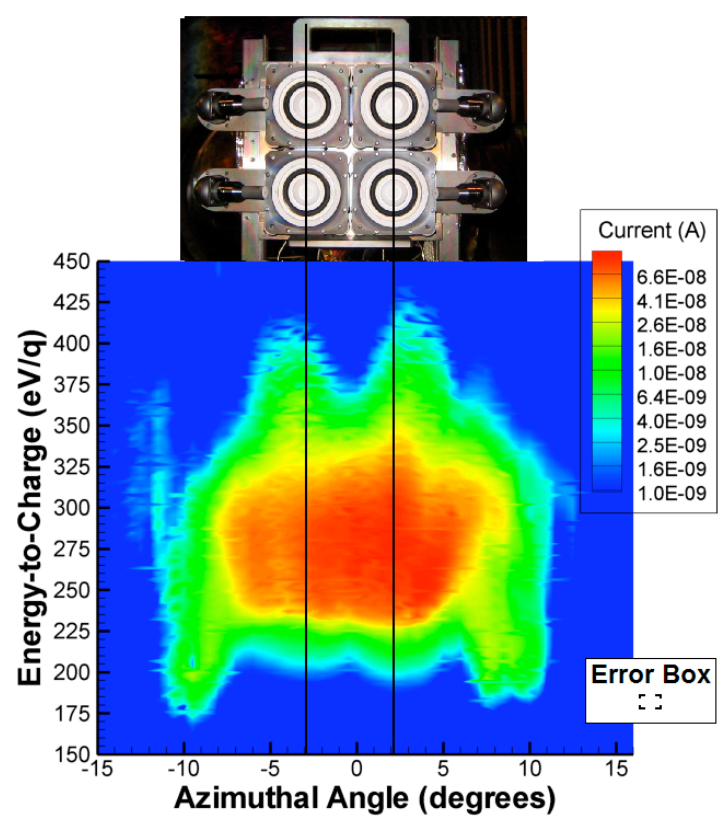

Figure 7. Current as a function of azimuthal angle and energy-to-charge for the BHT-600 cluster. The color coding is exponential to enhance low current features.

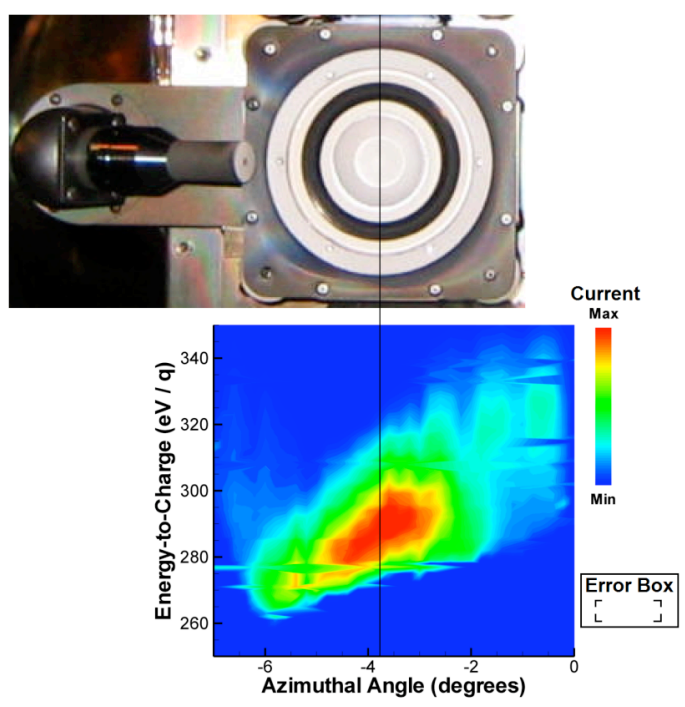

Figure 8. Current as a function of azimuthal angle and energy-to-charge ratio for single BHT-600 thruster operation along the cluster centerline. 
configuration of the P5 Hall thruster "twins." He found no change in the overall thrust of cluster configuration for the $5 \mathrm{~A}$ setting and a slight increase in the $10 \mathrm{~A}$ operation which he attributes to the increased ingestion of background particles due to facility effects. ${ }^{14}$ It is important to note that for the operation of the P5 cluster, the cathodes were not facing the discharge channels of the opposite thruster. However, for the BHT-600 cluster, this is the case, and measurements by TOPAZ support the theory that the crosspollination of electrons emitted by the cathodes could increase the overall ionization of beam ions, and therefore increase the overall thrust and thruster efficiency for the cluster configuration.

The asymmetry measured in Fig 8 also indicates the thruster could generate slight yawing-torque towards the cathode since ions are accelerated to higher energies away from the cathode versus near the cathode, however the relative flux of ions at different energies could affect the direction of the torque. Recent experiments conducted by Hofer suggest that a centrally placed cathode decreases plume divergence and results in an asymmetric plume about the thruster axis. ${ }^{22}$ Since a centrally-placed cathode would eliminate thruster output-power into the yawing-torque as predicted by TOPAZ, it is possible a slight increase in thrust force, and hence anode efficiency, would be measured with this configuration if the input power was held constant.

\section{Mass-per-Charge Distributions}

Figure 9 displays a plot of the mass- and energy-to-charge relationship for the cluster operation at the $0^{\circ}$ plume angle. Four peaks are visible in Fig. 9. The three on the right represent $\mathrm{Xe}^{3+}$, $\mathrm{Xe}^{2+}$, and $\mathrm{Xe}^{+}$, from left-to-right. However there is a peak at approximately $30 \mathrm{amu}$. Since $\mathrm{N}_{2}^{+}$has a mass-to-charge ratio of 28 $\mathrm{amu} / \mathrm{q}$ while $\mathrm{Xe}^{4+}$ has a ratio of $32.8 \mathrm{amu} / \mathrm{q}$, and both are within the mass-to-charge ratio resolution, the species is indiscernible. This measurement could also possibly be a combination of both species. Manzella has documented evidence of $\mathrm{N}_{2}{ }^{+}$emanating from the SPT100 Hall thruster, ${ }^{23}$ however King measured $\mathrm{N}^{+}$ions and negligible amounts of molecular-nitrogen ions. ${ }^{24}$ Since $\mathrm{Xe}^{4+}$ has been detected in Hall thruster plume in small quantities, ${ }^{25,26}$ it is likely this is the ion species; however this conclusion is taken with caution. The first

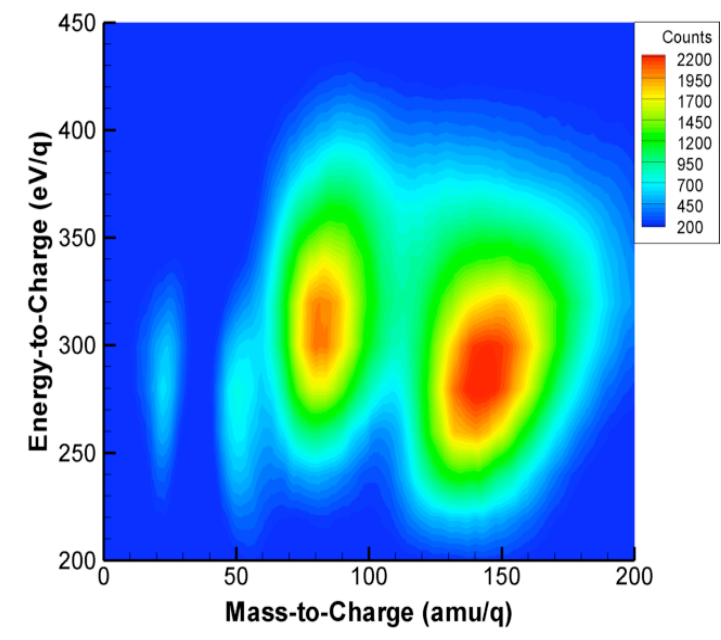

Figure 9. Energy-to-charge as a function of mass-per-charge for the cluster at a $0^{\circ}$ plume angle.

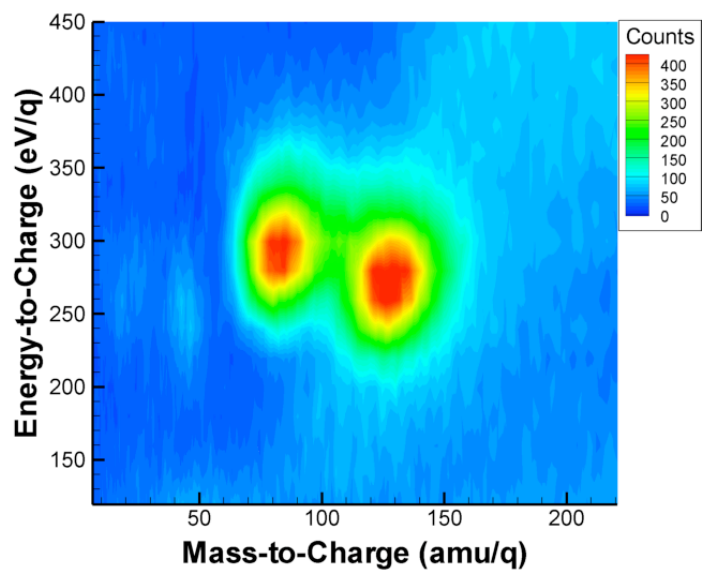

Figure 10. Energy-to-charge as a function of mass-per-charge for the cluster at a $20^{\circ}$ plume angle. three charge-states of Xenon, however, are easily recognized due to the large mass of xenon particles. The singly-charged and triply-charged xenon ions have peak energy-to-charge ratios at approximately $280 \mathrm{eV}$; however the doubly-charged xenon has a slightly higher energy-peak at $315 \mathrm{eV}$. The overall energy-to-charge distribution of the doubly-charged xenon is slightly higher as well. The likely cause is charge-exchange collisions which create particles with energy-to-charge ratios above the discharge voltage. Elastic collisions with other particles effectively broaden the energy distribution, since they allow for a variable amount of momentum-transfer (and therefore energy transfer) between the interacting particles.

A distribution of the energy-to-charge versus the mass-to-charge ratio for the $20^{\circ}$ plume angle is shown in Fig. 10. A decreased transmission is noticed overall, as indicated by the maximum number of counts (400) versus the $0^{\circ}$ (2200) and $10^{\circ}(1300)$ plume angles. This results in a slightly larger signal-to-noise ratio for the measurements. The plot clearly indicates large quantities of $\mathrm{Xe}^{+}$and $\mathrm{Xe}^{2+}$; however a very small quantity of triply-charged xenon is detected, and an almost imperceptible amount of possible $\mathrm{Xe}^{4+}$ is measured in the plume. This data agrees with previous measurements on the well-studied SPT-100 - significant quantities of multiply-charged ions are detected at plume angles within $20^{\circ}$, however, beyond this angle, the plume is composed of mostly singly-charged and some doubly-charged xenon ions. ${ }^{27}$ The energy distributions, however, are similar to the $0^{\circ}$ and $10^{\circ}$ plume angles. The $\mathrm{Xe}^{+}, \mathrm{Xe}^{2+}$, and $\mathrm{Xe}^{4+}$ ions have energy peaks slightly lower than the $\mathrm{Xe}^{2+}$ ions. As for the previous plume angles discussed, the $\mathrm{Xe}^{2+}$ could be more susceptible to CEX collisions in combination with momentum-exchange collisions resulting in an elevated energy distribution with respect to the other species of ions. 


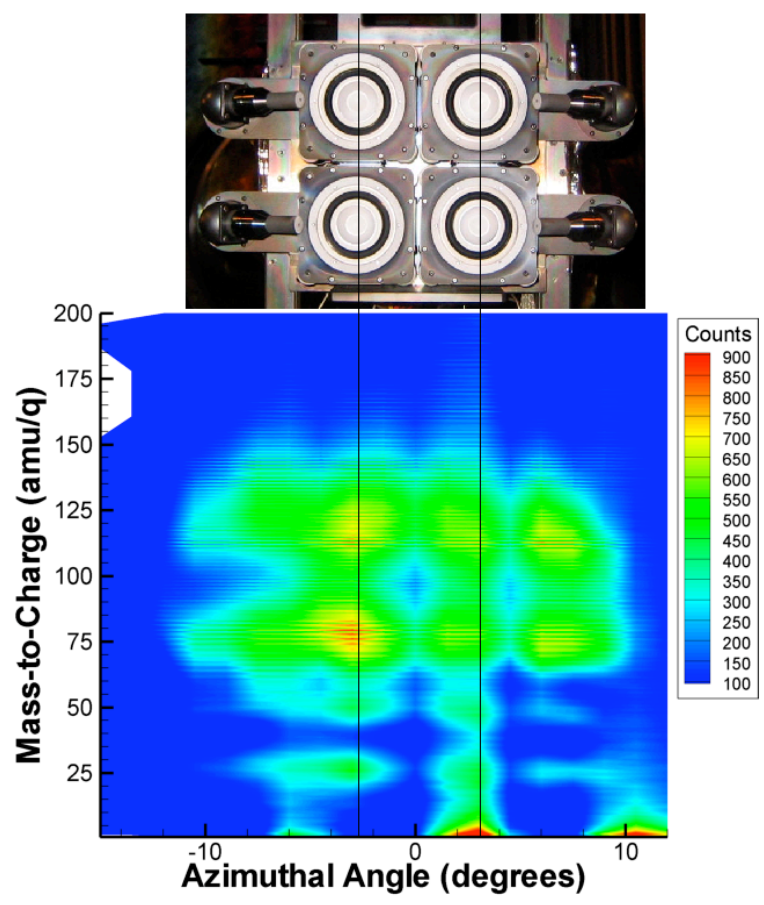

Figure 11. Mass-to-charge as a function of azimuthal angle for the cluster at a $0^{\circ}$ plume angle. The cluster is pictured for azimuthal-angle reference.

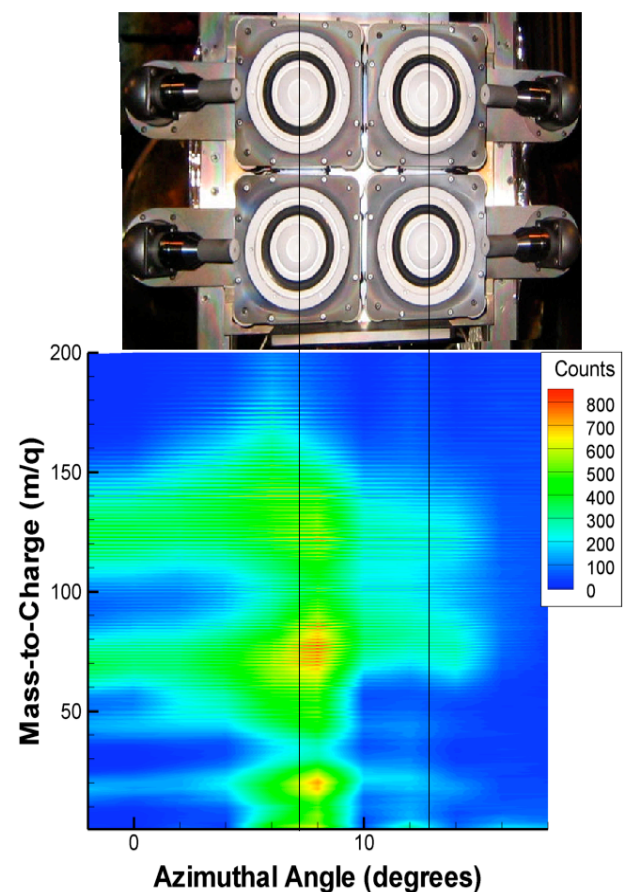

Figure 12. Mass-to-charge as a function of azimuthal angle for the cluster at a $10^{\circ}$ plume angle. The cluster is pictured (and scaled for a $10^{\circ}$ viewpoint) for azimuthal-angle reference.

Figure 12 displays a plot of the mass-per-charge relationship with azimuthal angle for the cluster operation at $0^{\circ}$. The azimuthal angle has been placed on the $x$-axis for correlation with the thruster positions. The rows in plots above each correspond with the different mass-per-charge species identified in the previous mass-per-charge profiles. It is evident that the lower mass-to-charge ions mostly emanate from the discharge channels while the singly and doubly charged ions are detected from all parts of the thruster. The measurements indicate almost an equal amount of $\mathrm{Xe}^{2+}$ as with $\mathrm{Xe}^{+}$, however there is a slight preference for the detection of smaller mass-to-charge ratio ions, since the have a larger "window of opportunity" to cross the aperture within the pulse width. This is accounted for in the calculation of ion species fractions in a later section. When comparing the Fig. 11 to the energyto-charge measurements with azimuthal on the cluster in Fig. 7, the energy-to-charge and mass-to-charge profiles can be compared with the emanating point of the thruster. The lower energy-to-charge $(175-300 \mathrm{eV})$ ions measured near the outsides of the cathodes correspond to singly- and doubly-charged ions, however along high energy peaks at the centerlines of the discharge channels, all charge states are measured.

Figure 12 shows a high dependence of which thrusters ions are detected from even at the slight plume angle of $10^{\circ}$. For this cluster plume angle, the left set of thrusters has an approximate individual-plume angle of $5^{\circ}$ while the right-two thrusters have an effective individual-plume angle of $15^{\circ}$. A higher amount of ions are detected from the left set of thrusters with the lower plume angle.

Unfortunately, the triply-ionized xenon ions blend in with the doubly-charged ions; however a higher detection rate of $\mathrm{Xe}^{2+}$ is measured at this slight angle. This agrees well with previous measurements on the SPT-100, for which measurements between $5-15^{\circ}$ off the thruster plume yield multiply-charged ions at higher amounts than singly charged xenon. ${ }^{24}$ Measurements from $9-11^{\circ}$ from one meter downstream show evidence of charge exchange from $\mathrm{Xe}^{4+} \rightarrow \mathrm{Xe}^{3+}$ and $\mathrm{Xe}^{3+} \rightarrow \mathrm{Xe}^{2+}$ at these angles for this thruster. More information the different types of CEX collisions can be found in Ref. 24. Large amounts of $\mathrm{Xe}^{4+} / \mathrm{N}_{2}{ }^{+}$are detected from the left-two thrusters which are near this angular range. This indicates multiply-charged ions tend to by slightly "off-focus" from the thruster plane, while singly- and doubly-charged xenon ions tend to emanate from much wider range of plume angles.

\section{Species Fractions}

A plot of the species fraction for the cluster operation at a $10^{\circ}$ plume angle shows some slight geometry dependence on the detection of the charge states in Fig. 13. Along the discharge channel centerlines, there is a slight 
dip in the $\mathrm{Xe}^{+}$and an increase in $\mathrm{Xe}^{2+}$. Over the left discharge channels, this coincides with the maximum $\mathrm{Xe}^{3+}$ fraction. As the previous measurements indicate, the production of multiply-charged ions occurs over the discharge-channel regions, and the fraction of single-charged xenon ions is lower. A possible reason is that the electrons are slightly more energetic near the discharge channel, and are therefore more likely to knock more electrons off propellant particles and create the multiple-charged ions in this region.

\section{Radial and Axial Velocity Distributions}

The following equations describe how the axial and radial velocity distributions for each species can be calculated through use of the azimuthal angle $\beta$, energy-to-charge $E / q$, and mass-to-charge $m / q$ :

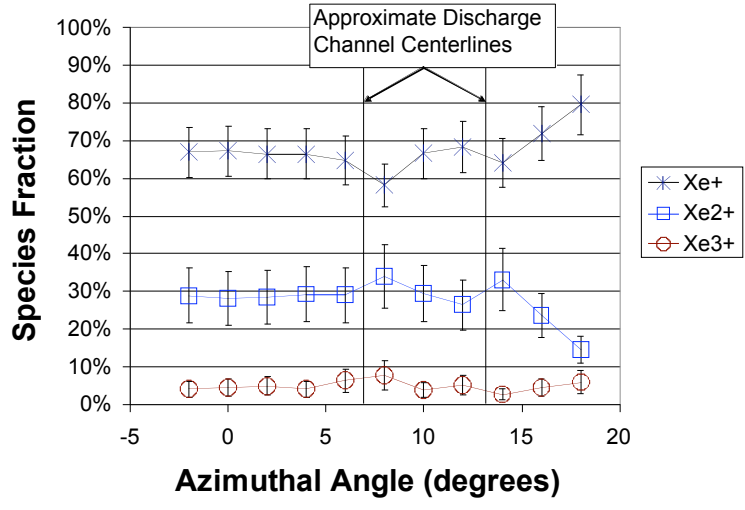

Figure 13. Species fraction as a function of azimuthal angle for the cluster operation at a $10^{\circ}$ plume angle.

$$
\begin{aligned}
& v_{\text {radial }}=-\sin (\beta) \sqrt{\frac{2(E / q)}{(m / q)}} \\
& v_{\text {axial }}=\cos (\beta) \sqrt{\frac{2(E / q)}{(m / q)}}
\end{aligned}
$$

The above calculations assume a rectilinear coordinate system. Ions traveling from the left side of the thruster with a negative azimuthal angular-trajectory have a positive radial velocity, whereas ions traveling from the right side of the thruster have a negative radial velocity. An ions moving away from the thruster has a positive axial velocity. The error bars in the first-, second-, and third-charge states are assumed to be $10 \%, 25 \%$, and $50 \%$, respectively.

A plot of the radial velocity is shown in Fig. 14. The plot is approximately symmetric (as expected) about 0 $\mathrm{km} / \mathrm{s}$. This is also the most likely radial velocity - ions moving perpendicular to the plane of the discharge channels. However two spikes are measured on each side. It is likely these correspond with measurements of ions from the left and right regions of the discharge channel near the cathodes. Since the $\mathrm{Xe}^{2+}$ and $\mathrm{Xe}^{3+}$ ions were more likely to emanate from the discharge channel centerlines (which is $2.3^{\circ}$ off from the cluster centerline), they are less likely to be detected from the thruster centerline. The second- and third-charge xenon ions have similar profiles, although (as shown below) $\mathrm{Xe}^{3+}$, has a much higher axial velocity. This indicates the trajectory of $\mathrm{Xe}^{3+}$ is at slighter azimuthal angles than the doubly-charged ions, allowing for both species to have similar radial velocity distributions.

Figure 15 displays a plot of the axial velocity for each species. Peaks with successively higher axial velocities were measured for the first, second, and third charge-states of xenon. However, it is interesting to note the double peak that is present for $\mathrm{Xe}^{+}$. The likely reason for this is the existence of a strong focusing effect for $\mathrm{Xe}^{+}$

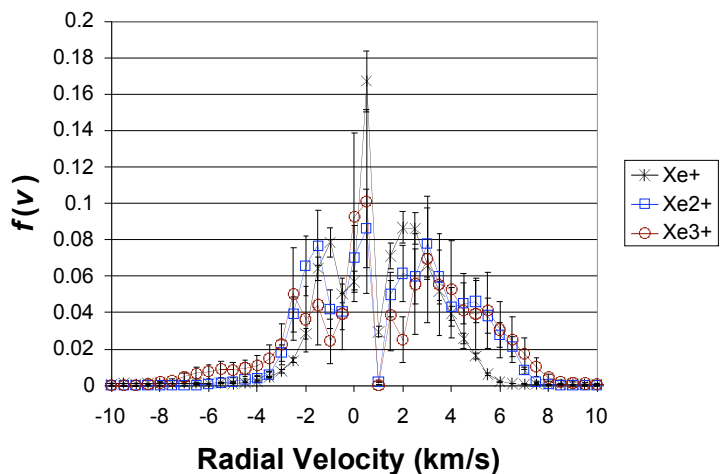

Figure 14. Radial velocity distribution of $\mathrm{Xe}^{+}$, $\mathrm{Xe}^{2+}$, and $\mathrm{Xe}^{3+}$ from the $0^{\circ}$ plume angle for the cluster operating condition.

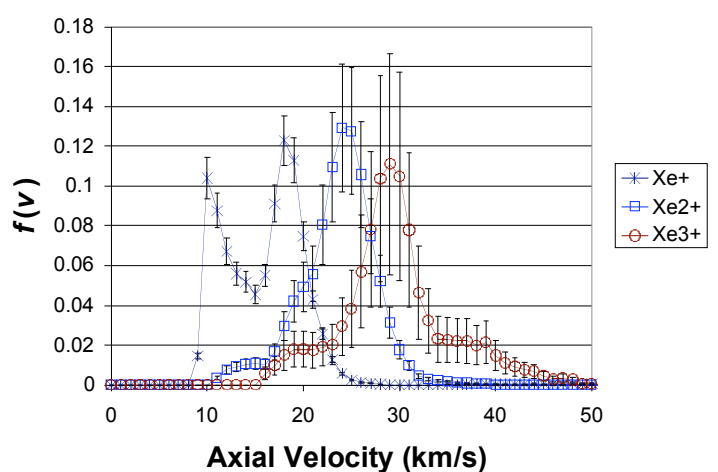

Figure 15. Axial velocity distribution of $\mathrm{Xe}^{+}, \mathrm{Xe}^{2+}$, and $\mathrm{Xe}^{3+}$ from the $0^{\circ}$ plume angle for the cluster operating condition. 
emanating from the interior regions (near the cluster centerline) of the thruster. These ions have little radial velocity, and are accelerated in a mostly axial direction. This accounts for the higher peak for $\mathrm{Xe}^{+}$. Singly-charged xenon ions that are detected from near the cathode, however, have lower energies as shown in the previous chapter. Since the angle of these ions also induces "cosine-losses" in the axial direction, they have significantly lower axial velocities. The single-charged xenon ions emanating from regions near the cathodes from either side of the cluster most likely contributes to the lower peak in the velocity distribution. The $\mathrm{Xe}^{2+}$ and $\mathrm{Xe}^{3+}$ ions, however, are generated from near the discharge channel regions, and not as much from the outskirts of the cluster. Therefore, the axial velocity is not decreased by "cosine-losses" and near-cathode effects.

\section{Acknowledgments}

The authors greatly acknowledge Dr. Stefan Scherer for the lease of the channel electron multiplier and power supply equipment as well as use of the ion accelerator and vacuum chamber for the characterization of TOPAZ. The authors thank Chuck Navarre and Robb Gillespie for their highly precise machining of TOPAZ and Robert Lobbia for the setup and operation of the Busek BHT-600 Hall thruster cluster. Finally, the authors thank Robert Lundgren for offering his expertise on the design of TOPAZ. A. Victor was supported by the NASA-funded Michigan Space Grant Consortium as well as the Rackham Graduate School at the University of Michigan.

\section{References}

${ }^{1}$ Haas, J. M., Gulczinski, F. S., Gallimore, A. D., Spanjers, G. G., et al., "Performance Characteristics of a 5 kW Laboratory Hall Thruster," AIAA-1998-3503, 34th AIAA/ASME/SAE/ASEE Joint Propulsion Conference \& Exhibit, Cleveland, OH, July 12-15, 1998.

${ }^{2}$ Spores, R. A., Spanjers, G.G., Birkan, M., Lawrence, T.J., "Overview of the USAF Electric Propulsion Program," AIAA-2001-3225, 37th AIAA/ASME/SAE/ASEE Joint Propulsion Conference and Exhibit, Salt Lake City, UT, July 8-11, 2001.

${ }^{3}$ Dunning, J., Sankovic, J., "NASA's Electric Propulsion Program," AIAA-2000-3145, 36th

AIAA/ASME/SAE/ASEE Joint Propulsion Conference \& Exhibit, Huntsville, AL, July 17-19, 2000.

${ }^{4}$ Manzella, D., Jankovsky, R., Hofer, R., "Laboratory Model 50 kW Hall Thruster," AIAA-2002-3676, 38th

AIAA/ASME/SAE/ASEE Joint Propulsion Conference and Exhibit, Indianapolis, Indiana, July 7-10, 2002.

${ }_{5}^{5}$ Jacobson, D. T., Jankovsky, R. S., Rawlin, V. K., Manzella, D. H., "High Voltage TAL Performance," AIAA2001-3777, 37th AIAA/ASME/SAE/ASEE Joint Propulsion Conference \& Exhibit, Salt Lake City, UT, July 8-11, 2001.

${ }^{6}$ Gallimore, A. D., "Near- and Far-Field Characterization of Stationary Plasma Thruster Plumes," Journal of Spacecraft and Rockets, Vol. 38, No. 3, pp. 441-453, May-June 2001.

${ }^{7}$ Victor, A. L., Zurbuchen, T. H., Gallimore, A. D., "The Top Hat Electric Propulsion Plume Analyzer (TOPAZ): Preliminary Data on the BHT-600 Cluster," AIAA-2005-3870, 41st AIAA/ASME/SAE/ASEE Joint Propulsion Conference, Tucson, AZ, July 10-13, 2005.

${ }^{8}$ Bame, S. J., McComas, D. J., Young, D. T., Belian, R. D., "Diagnostics of Space Plasmas," Review of Scientific Instruments, Vol. 57, No. 8, pp. 1711-1716, August 1986.

${ }^{9}$ Vilppola, J. H., Tanskanen, P. J., Huomo, H., Barraclough, B. L., "Simulations of the Response Function of a Plasma Ion Beam Spectrometer for the Cassini Mission to Saturn," Review of Scientific Instruments, Vol. 67, No. 4, pp. 1494-1501, April 1996.

${ }^{10}$ Hofer, R. R., Haas, J. M., Gallimore, A. D., "Ion Voltage Diagnostics in the Far-Field Plume of a HighSpecific Impulse Hall Thruster," AIAA-2003-5162, 39th AIAA/ASME/SAE/ASEE Joint Propulsion Conference \& Exhibit, Huntsville, AL, July 20-23, 2003.

${ }^{11}$ Herman, D. A., Gallimore, A. D., "Comparison of Discharge Plasma Parameters in a 30-cm NSTAR Type Ion Engine with and without Beam Extraction," AIAA-2003-5162, 39th AIAA/ASME/SAE/ASEE Joint Propulsion Conference \& Exhibit, Huntsville, AL, July 20-23, 2003.

${ }^{12}$ Book, D. L., NRL Plasma Formulary, Naval Research Laboratory, Washington, DC, 2001.

${ }^{13}$ Young, D. T., Measurement Techniques in Space Plasmas: Particles, Vol. 102, American Geophysical Union, Washington, DC, 1998.

${ }^{14}$ Walker, M. L. R., Victor, A. L., Hofer, R. R., Gallimore, A. D., "Effect of Backpressure on Ion Current Density Measurements in Hall Thruster Plumes," Journal of Propulsion and Power, Vol. 21, No. 3, pp. 408-415, May-June 2005.

${ }^{15}$ Hofer, R. R., Gallimore, A. D., "Efficiency Analysis of a High-Specific Impulse Hall Thruster," AIAA-20043602, 40th AIAA/ASME/SAE/ASEE Joint Propulsion Conference, Fort Lauderdale, FL, July 11-14, 2004. 
${ }^{16}$ Victor, A. L., Zurbuchen, T. H., Gallimore, A. D., "Top Hat Electrostatic Analyzer for Far-Field Electric Propulsion Plume Diagnostics," Review of Scientific Instruments, Vol. 77, No. 1,

${ }^{17}$ Oleson, S., Elliott, F., "The Electric Propulsion Segment of Prometheus 1," AIAA-2005-3888, 41st

AIAA/ASME/SAE/ASEE Joint Propulsion Conference and Exhibit, Tucson, AZ, July 10-13, 2005.

${ }^{18}$ Carlson, C. W., McFadden, J. P., Design and Application of Imaging Plasma Instruments, Vol. 102, American Geophysical Union, Washington, DC, 1998.

${ }^{19}$ Victor, A. L., Zurbuchen, T. H., Gallimore, A. D., "Development of the Top Hat Electric Propulsion Plume Analyzer (TOPAZ): Mass Analyzer Design and Preliminary Calibration Data," IEPC-2005-016, 29th International Electric Propulsion Conference, Princeton, NJ, October 31-November 4th, 2005.

${ }^{20}$ Dushman, S., Vol. 4, John Wiley \& Sons, Inc., New York, NY, 1958.

${ }^{21}$ Beal, B. E., "Clustering of Hall Effect Thrusters for High-Power Electric Propulsion Applications," Ph.D. Thesis,Dept. of Aerospace Engineering, University of Michigan, Ann Arbor, MI, 2004.

${ }^{22}$ Hofer, R. R., Johnson, L. K., Goebel, D. M., Fitzgerald, D. J., "Effects of an Internally-Mounted Cathode on Hall Thruster Plume Properties," AIAA-2006-4482, 42nd AIAA/ASME/SAE/ASEE Joint Propulsion Conference \& Exhibit, Sacramento, CA, July 9-12, 2006.

${ }^{23}$ Manzella, D. H., "Stationary Plasma Thruster Plume Emissions," IEPC-93-097, 23rd International Electric Propulsion Conference, Seattle, WA, September 13-16, 1993.

${ }^{24}$ King, L. B., "Transport Property and Mass Spectral Measurements in the Plasma Exhaust Plume of a Halleffect Space Propulsion System," Thesis, Dept. of Aerospace Engineering, University of Michigan, Ann Arbor, MI, 1998.

${ }^{25}$ Linnell, J. A., Gallimore, A. D., "Efficiency Analysis of a Hall Thruster Operating with Krypton and Xenon," AIAA-2005-3683, 41 st AIAA/ASME/SAE/ASEE Joint Propulsion Conference and Exhibit, Tucson, AZ, July 1013.

${ }^{26}$ Kim, S.-W., Gallimore, A. D., "Plume Study of a 1.35 kW SPT-100 Using an ExB Probe," AIAA-99-2423, 35th AIAA/ASME/SAE/ASEE Joint Propulsion Conference, Los Angeles, CA, June 20-23, 1999.

${ }^{27}$ Manzella, D. H., "Stationary Plasma Thruster Plume Emissions," IEPC-93-097, 23rd International Electric Propulsion Conference, Seattle, WA, September 13-16, 1993. 